do a biopsy. So, we tracked some patients with colon ulcer in order to know the etiology of these colon ulcers.

Methods A total of 32 cases were enrolled. The mean age was (46.3 \pm 14.8 ) years old (range, $21-70$ years). The ratio of male to female was 9:7. The main reasons for patients.

Undergoing colonoscopy were abdominal pain, diarrhea, and bloody purulent stool. The symptoms lasted for at least a month or more. The basic information, medical history, clinical manifestations, computed tomography (CT), endoscopy of the patients and pathological examination were analyzed.

Results The 32 patients' colonoscopy showed isolate or multiple colon ulcer. At the same time, we did a biopsy. The histopathology showed mucosal chronic inflammation. Combined with the detailed medical history, lab examination, abdominal CT and colonoscopy, we made the initial diagnosis. $8(25 \%)$ were NSAIDs-related colonic ulcer (figure 1). 6 $(18.8 \%)$ were ischemic bowel disease. 3(9.4\%) were allergic purpura. 2(6.3\%) were Behcet' disease. 1(3.1\%) was Wegener's granulomatosis (figure 2). There are 12 cases, we did not find a clear etiology of colonic ulcer. We did colonoscopy and multi-slice biopsy for all the patients after 3 months. The 20 patients, who had an initial diagnosis, the colon ulcer changed small even disappear after treatment. The other 12 patients, who had no initial diagnosis without any treatment, 6 of them had no changes in the lesions. The histopathology showed 3 were tuberculosis, 2 developed typical ulcerative colitis, 1 was lymphoma. The ulcerations of the other 3 with no initial diagnosis changed small. But the histopathology showed nonspecific mucosal chronic inflammation. The remaining 3, the lesions had disappeared. The follow-up continues.

Conclusions There are various manifestations of colonic ulcer with colonoscopy. A detailed medical history is helpful for our diagnosis. The follow-up is important for no reason colonic ulcer. We should not diagnose and use medicine blindly.

\section{IDDF2020-ABS-0216 CLINICAL ANALYSIS OF SURGICAL INTERVENTION IN THE TREATMENT OF T4 COLORECTAL CANCER WITH PERITONEAL CARCINOMATOSIS}

${ }^{1}$ Wei Chen*, ${ }^{2}$ Xiang Peng, ${ }^{2}$ Min Zhi. ${ }^{1}$ Department of Colorectal Surgery, Guangdong Provincial Key Laboratory of Colorectal and Pelvic Floor Disease, the Sixth Affiliated Hospital, Sun Yat-sen University, China; ${ }^{2}$ Gastroenterology, Guangdong Provincial Key Laboratory of Colorectal and Pelvic Floor Disease, the Sixth Affiliated Hospital, Sun Yat-sen University, China

\subsection{6/gutjpl-2020-IDDF.126}

Background To observe the factors related to T4 patients who underwent surgery for colorectal peritoneal carcinomatosis.

Methods The clinical and follow-up data of 154 T4 colorectal cancer patients who underwent surgery in the first Affiliated Hospital of Sun Yat-sen University between August, 1994 and December, 2005. The influence of surgery on the prognosis was evaluated.

Results At the end of December of 2010 or death, the overall survival time was 91.7 months. The median survival time of complete cytoreduction group, the incomplete cytoreduction and the palliative surgery group were 102.9, 18.8 and 19.7 months, respectively. The complete cytoreduction had significant survival benefit than the palliative surgery group. Univariate analysis showed that the location, histological grade, complete cytoreduction and liver metastasis were associated with OS $(\mathrm{P}<0.05)$. In addition, Cox multivariate analysis showed that the complete cytoreduction and liver metastasis were independent factors influencing patient survival.

Conclusions Patients performed completed cytoreduction have a relatively good prognosis. Compared with palliative surgery, the incomplete cytoreduction fails to improve patient prognosis.

\section{IDDF2020-ABS-0217 CLINICAL MANIFESTATION AND BACTERIA OF SECONDARY SEPSIS FROM HEPATIC DISEASE AND CHOLESTASIS}

${ }^{1}$ Le Thi Thu Hien*, ${ }^{2}$ Le Quoc Tuan, ${ }^{3}$ Nguyen Quang Duat. 'Department of Internal Medicine, Thai Nguyen University of Medicine and Pharmacy, Vietnam; ${ }^{2}$ Department of Internal Medicine, Thanh Ba Hospital, Vietnam; ${ }^{3}$ Department of Internal Medicine, 103 Military Hospital, Vietnam

\subsection{6/gutjpl-2020-IDDF.127}

Background Biliary stasis and increased intraductal pressure are central to the pathogenesis of acute cholangitis. Biliary stasis inhibits the continuous flushing activity of bile and the bacteriostatic effect of bile salts, which help maintain bile sterility. Elevated intraductal pressure favors translocation of bacteria and toxins out of the ducts and into the systemic circulation, which can result in sepsis. Sepsis is among the most common causes of mortality for hospitalized patients worldwide, and its incidence is steadily increasing.

Aims To characterize clinical manifestation and identify bacteria of secondary sepsis from hepatic disease and cholestasis.

Methods We conducted a prospective study of 38 patients with secondary sepsis from hepatic disease and cholestasis at the 108 Military Central Hospital from January 2018 to August 2019. Contributions of the history, physical examination, laboratory investigation and subclinical in making medical diagnoses. PCR-based Sepsis@Quick test is for identification of sepsis-causative pathogens.

Results On examination: fever (100\%); abdominal pain $(39.5 \%)$ and jaundice (100\%). Laboratory investigation: White blood cell $(16.98 \pm 8.46 \mathrm{G} / \mathrm{L})$; Neutrophil (85.3 \pm 10.82\%); Procalcitonin $(21.18 \pm 27.16 \mathrm{ng} / \mathrm{ml})$. Procalcitonin values demonstrated a statistically significant and directly proportionate relationship to severity of sepsis. Secondary sepsis from cholestasis: biliary tract cancer 28.9\%; gallstone 28.9\%; pancreatic cancer $10.5 \%$. Secondary sepsis from hepatic diseases: hepatocellular carcinoma $13.2 \%$; cirrhosis $10.5 \%$; liver abscess 7.9\%. Sepsis-causative pathogens: Escherichia coli 55.3\%; Klebsiella pneumoniae 31.6\%; Citrobacter koseri 2.6\%; Streptococcus anginosus 2.6\%; Enterococcus faecalis 2.6\%; Citrobacter freundii 2.6\%; Klebsiella oxytoca 2.6\%; Pseudomonas aeruginosa $2.6 \%$.

Conclusions Etiology of secondary sepsis from hepatic disease and cholestasis the most is Escherichia coli, second is Klebsiella pneumoniae, of these we find Escherichia coli most commonly in gallstones, Klebsiella pneumonia most commonly in cholangiocarcinoma. 